\title{
BI-ORDERINGS ON PURE BRAIDED THOMPSON'S GROUPS
}

\author{
JOSÉ BURILLO AND JUAN GONZÁLEZ-MENESES
}

\begin{abstract}
In this paper it is proved that the pure braided Thompson's group $B F$ admits a bi-order, analog to the bi-order of the pure braid groups.
\end{abstract}

\section{INTRODUCTION}

Braid groups have been a constant object of study all along the 20th century, and have exerted a strong fascination on algebraists since their inception, due to their rich and interesting properties and useful applications to other branches of mathematics. Literature about braid groups is abundant; we will mention only a small sample of it, namely, the seminal work of Artin [1], a more modern text [11], and a recent survey [3]. In particular, the properties which will be of interest here are their orderings and bi-orderings, see [8], [10] and [12] for details. Orderings of groups are also a classical subject of study, related, for instance, to the existence of zero divisors in the group ring.

Thompson's groups have been studied since the late 1960s, initially as examples of infinite, finitely presented simple groups $-V$ being the first such a group known-, but in the subsequent years they showed other, equally striking properties, for instance, $F$ was the first torsion-free $F P_{\infty}$ group, see [6]. For details and many proofs of the properties of Thompson's groups see the excellent introduction in [7].

Recently and independently, Brin [5] and Dehornoy [9] have introduced braided versions of Thompson's groups, which show a mixture of properties of both. They are a very natural extension of Thompson's group $V$, where the permutations, characteristic in $V$, have been replaced by braids. Hence, the braided Thompson's group $B V$ (in Brin's notation) is a torsion-free version of $V$, sharing many of its properties, for instance, finite presentation, with a presentation very similar to that of $V$. One can think of the braided Thompson's groups as "Artin" versions of Thompson's groups $V$ and $F$, very much in the same way as braid groups are the corresponding Artin groups for the permutation groups.

The main concern of this paper are some properties that braided Thompson's groups inherit from braid groups, namely, orderings and bi-orderings. It is a well-known fact that braid groups are orderable but not bi-orderable, and pure braid groups are bi-orderable. The same thing happens in the braided Thompson's groups: the braided version of $V$, analog to $B_{\infty}$, is orderable but not bi-orderable, and its pure subgroup $B F$ is bi-orderable, as it models on $P_{\infty}$. This last statement is the main result of this paper, Theorem 3.3. On the way to proving this result, we study a somewhat non-standard version of the pure braid group on infinitely many strands, where there are $n$ different embeddings of $P_{n}$ into $P_{n+1}$, each one defined by splitting a given strand in two. The direct limit obtained

Date: April 26, 2007.

The first author acknowledges support from grant MTM2005-04104.

The second author was partially supported by MTM2004-07203-C02-01 and FEDER. 
this way is an infinitely generated group, already appeared in [4], and which is also biorderable, a fact whose proof is the crucial ingredient of the proof of the main result, and takes up the largest and most technical part of Section 3. The previous sections of the paper are dedicated to set up the table for the main result, the first section with an introduction to the braided Thompson's groups, and the second one about orderings and the ordered groups that will be involved in the construction of the bi-order of $B F$.

The first author would like to acknowledge the great hospitality of the Departamento de Álgebra of the Universidad de Sevilla, during a visit to which this work was developed.

\section{The BRAided Thompson's GROUps}

Thompson's group $F$ is the group of piecewise-linear, orientation-preserving homeomorphisms of the interval $[0,1]$ whose breakpoints are dyadic integers, and whose slopes are powers of 2. An element of $F$ can be understood as a map between two subdivisions of the unit interval, subdivisions into intervals of lengths $1 / 2^{n}$ with dyadic endpoints, where the subintervals are mapped linearly in order-preserving fashion. See [7] for an excellent introduction to Thompson's groups and proofs of their basic properties.

Such a subdivision of the unit interval is in correspondence with a binary tree, in the usual way. Hence, an element of $F$ can be represented as a pair of binary trees $\left(T_{-}, T_{+}\right)$, with the same number of leaves, as is shown in Figure 1. An element admits more than one representation as a pair of binary trees by further subdividing an interval and its target, but each element admits a unique reduced element whose subdivision is optimal. See again [7] for details.
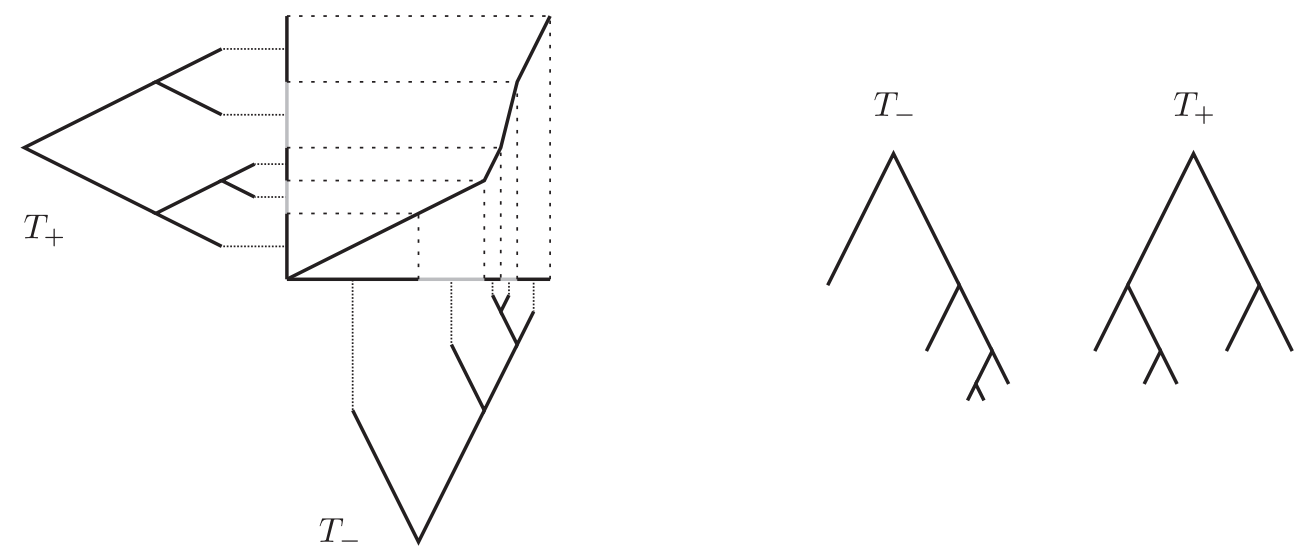

Figure 1. An element of $F$, and its reduced pair of binary trees $\left(T_{-}, T_{+}\right)$.

Thompson's group $V$ is represented in a similar way by subdivisions of the interval, but now the subintervals are still mapped linearly to each other, but they can be permuted, thus not necessarily preserving their order. So an element of $V$ is represented as a triple $\left(T_{-}, \pi, T_{+}\right)$, where the binary trees $T_{-}$and $T_{+}$have $n$ leaves, and $\pi$ is a permutation in $S_{n}$ which indicates how the leaves are mapped to each other. See [7] and Figure 2 to understand this interpretation of elements of $V$.

The group $V$ is infinite, simple and finitely presented, being the first such group historically known. Observe that fixing a tree $T$, and varying $\pi$, the elements $(T, \pi, T)$ form a subgroup 

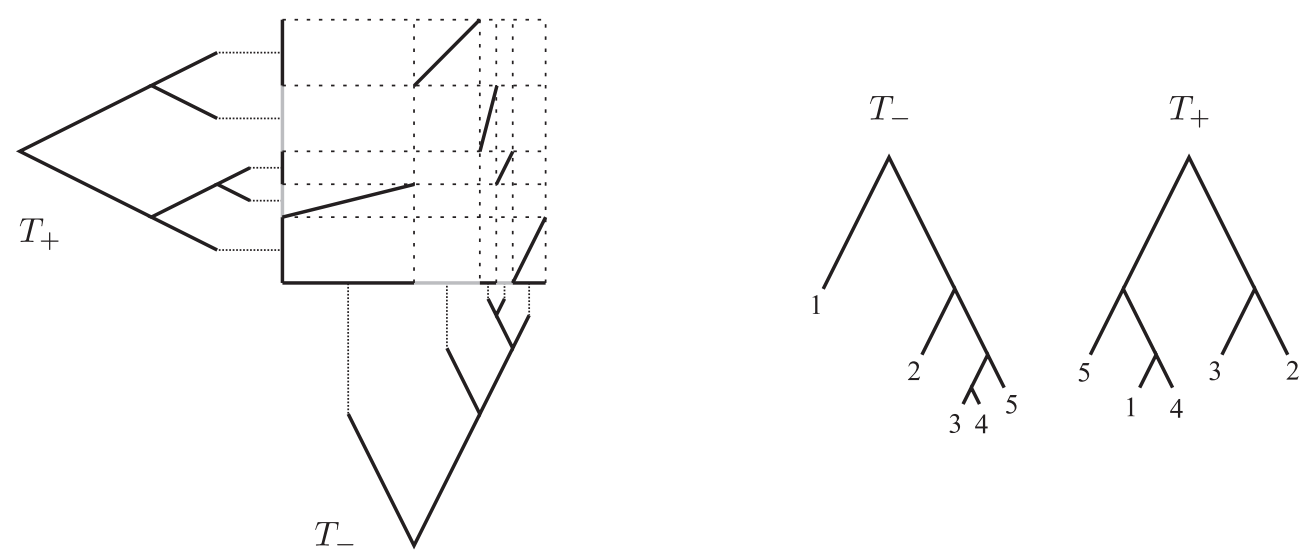

Figure 2. An element of $V$, and its pair of binary trees $\left(T_{-}, T_{+}\right)$. The labels of the leaves describe the permutation $\pi$.

of $V$ isomorphic to $S_{n}$. Hence, $V$ has torsion, and indeed, it contains all finite groups as subgroups. As a contrast, $F$ is torsion-free.

We will denote Artin's braid group in $n$ strands by $B_{n}$. It is the fundamental group of the configuration space of $n$ unordered points in the plane. See [2] for details. Its elements are usually visualized as a set of $n$ disjoint strands in 3-space, as in the left hand side of Figure 3, and it admits the following well-known presentation [1]:

$$
B_{n}=\left\langle\begin{array}{l|ll}
\sigma_{1}, \ldots, \sigma_{n-1} & \begin{array}{l}
\sigma_{i} \sigma_{j}=\sigma_{j} \sigma_{i} \\
\sigma_{i} \sigma_{j} \sigma_{i}=\sigma_{j} \sigma_{i} \sigma_{j}
\end{array} \text { if }|i-j|=1 .
\end{array}\right\} .
$$

Let

$$
\rho_{n}: B_{n} \longrightarrow S_{n}
$$

be the homomorphism from the braid group onto the symmetric group, which maps a braid to the permutation it induces on the base points. The kernel of $\rho_{n}$ is $P_{n}$, the subgroup of pure braids in $n$ strands.
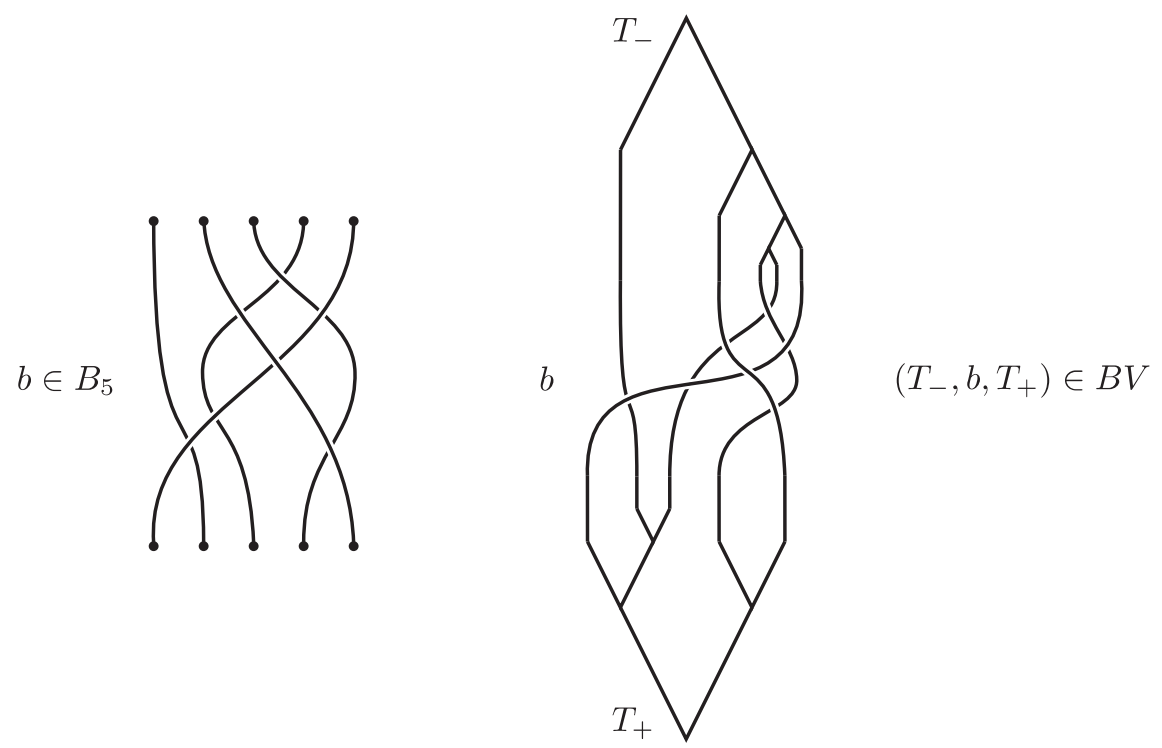

Figure 3. An element of $B_{5}$ and an element of $B V$. 
The braided group $B V$ (which means "braided $V$ ") is a torsion-free version of $V$ using braids instead of permutations. Elements of $B V$ are seen as triples $\left(T_{-}, b, T_{+}\right)$, where the trees $T_{-}$and $T_{+}$have $n$ leaves, and $b \in B_{n}$ is a braid in $n$ strands. The braid is understood as joining the leaves of $T_{-}$with those of $T_{+}$, see the right hand side of Figure 3 for an example. An element of $B V$, as it happens in other Thompson's groups, admits many representatives, by adding carets to the trees and splitting the corresponding strands. Hence, to multiply two elements in $B V$ we only need to subdivide the trees until we find representatives with matching trees: given two elements $\left(T_{-}, b, T_{+}\right)$and $\left(T_{-}^{\prime}, b^{\prime}, T_{+}^{\prime}\right)$, we construct, by adding carets to the trees and splitting strands into parallel ones, two representatives $\left(\bar{T}_{-}, \bar{b}, \bar{T}_{+}\right)$and $\left(\bar{T}_{-}^{\prime}, \bar{b}^{\prime}, \bar{T}_{+}^{\prime}\right)$ such that $\bar{T}_{+}=\bar{T}_{-}^{\prime}$, and then the product is the triple $\left(\bar{T}_{-}, \overline{b b}^{\prime}, \bar{T}_{+}^{\prime}\right)$, where the product of the two braids takes place in the corresponding braid group, with as many strands as leaves in the trees. Observe that since $\bar{T}_{+}=\bar{T}_{-}^{\prime}$, the two braids have the same number of strands. For details on $B V$, see [5], [9] and [4].

As it happens in $B_{n}$, forgetting the braid and focusing on the corresponding permutation gives a homomorphism

$$
\widetilde{\rho}: B V \longrightarrow V
$$

such that

$$
\widetilde{\rho}\left(T_{-}, b, T_{+}\right)=\left(T_{-}, \rho(b), T_{+}\right) .
$$

Notice that the element in Figure 3 maps to the element in Figure 2.

Finally, the group $B F$ is the subgroup of $B V$ of those elements $\left(T_{-}, p, T_{+}\right)$whose braid is pure. See an example in the left hand side of Figure 4. Observe that if $p$ is pure, then $\rho(p)$ is the identity permutation, so then $\left(T_{-}, \rho(p), T_{+}\right)$is actually an element of $F$ inside $V$. Then, it is clear that $B F=\widetilde{\rho}^{-1}(F)$, hence the name $B F$ ("braided $F$ "). For details on $B F$, in particular for a finite presentation, see [4].

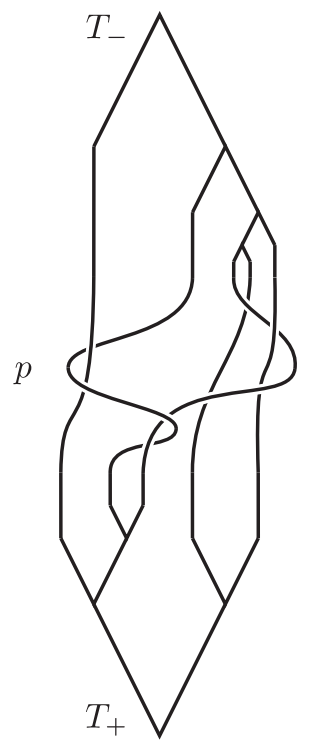

$x=\left(T_{-}, p, T_{+}\right) \in B F$

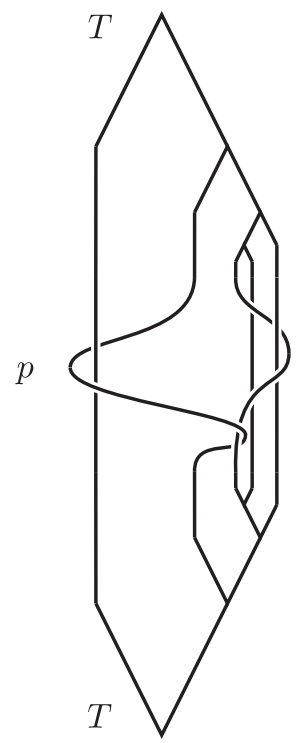

$y=(T, p, T) \in P B V$

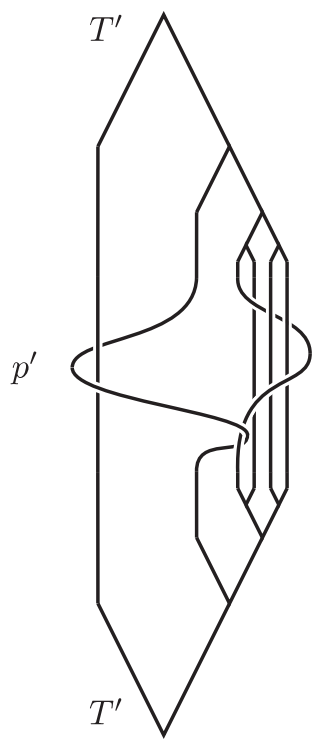

$y=\left(T^{\prime}, p^{\prime}, T^{\prime}\right) \in P B V$

Figure 4. An element $x \in B F$, and an element $y \in P B V$ represented in two different ways. 
A group which will be of great relevance later is the group $P B V=\operatorname{ker} \widetilde{\rho}$. Clearly, since $\left(T_{-}, p, T_{+}\right)$is in $P B V$ if $\widetilde{\rho}\left(T_{-}, p, T_{+}\right)=1$, we must have that $p$ is a pure braid, and also $T_{-}=T_{+}$. Then, $P B V$ is the subgroup of $B V$ (actually inside $B F$ ) of all those elements where the two trees are the same and the braid is pure. See an example in Figure 4. Observe that if an element has a representative where the two trees are the same and the braid is pure, then all representatives satisfy these two conditions.

Given a tree $T$ with $n$ leaves, the subgroup of $P B V$ of the elements represented by $(T, p, T)$, varying $p$, is a group isomorphic to $P_{n}$. We will denote this particular copy of $P_{n}$ inside $P B V$ by $P_{n, T}$, and there are as many such subgroups isomorphic to $P_{n}$ inside $P B V$ as there are trees with $n$ leaves, i.e., the Catalan number

$$
\operatorname{Cat}(n-1)=\frac{1}{n}\left(\begin{array}{c}
2 n-2 \\
n-1
\end{array}\right)
$$

If we now consider the tree $T^{\prime}$ obtained from $T$ by attaching a caret to the $i$-th leaf of $T$, we get another representative for $(T, p, T)$, namely, $\left(T^{\prime}, p^{\prime}, T^{\prime}\right)$, where $p^{\prime}$ has been obtained from $p$ by splitting the $i$-th strand in two parallel ones (see examples in Figure 4 and Figure 5). We have then a one-to-one homomorphism

$$
\alpha_{n, T, i}: P_{n, T} \longrightarrow P_{n+1, T^{\prime}}
$$

obtained via this process. The group $P_{n, T}$ can be identified with the subgroup of $P_{n+1, T^{\prime}}$ of those elements whose $i$-th and $(i+1)$-st strands are parallel. Observe that both an element of $P_{n, T}$ and its image under $\alpha_{n, T, i}$ represent the same element in $P B V$. With these subgroups and maps, the following proposition is straightforward.
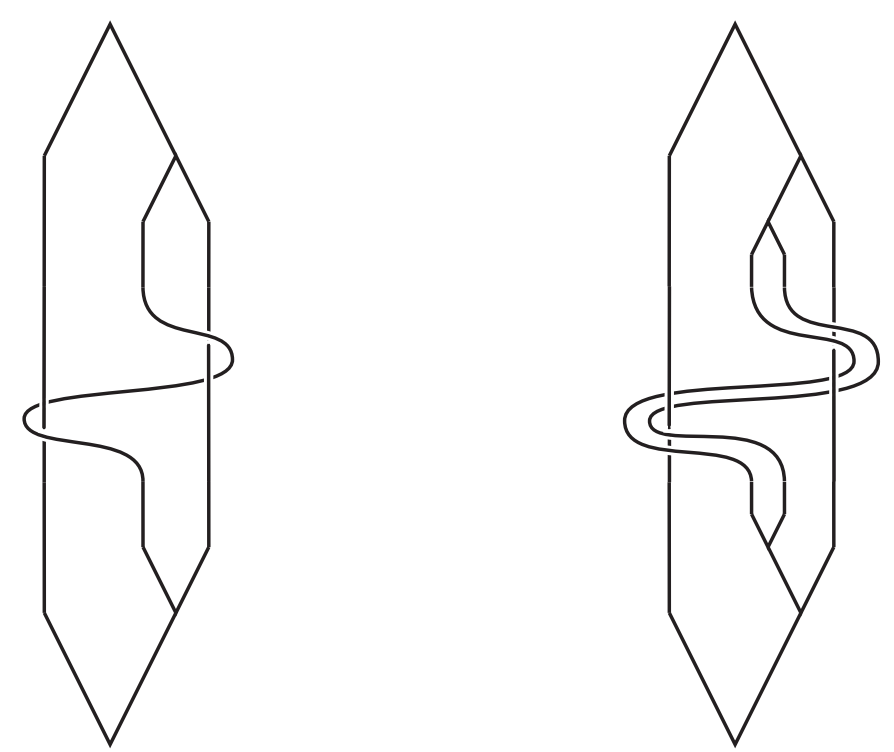

Figure 5. An example of an element in $P_{3, T}$ and its image under $\alpha_{3, T, 2}$, by splitting the second strand.

Proposition 1.1. The groups $P_{n, T}$, for $n>1$ and $T$ a tree with $n$ leaves, together with the maps $\alpha_{n, T, i}$, for $1 \leq i \leq n$, form a direct system of groups and homomorphisms, whose direct limit is isomorphic to PBV. That is,

$$
P B V=\underset{T}{\lim } P_{n, T}
$$


In the next section we will recall the notion of orderable groups. We will also recall that the pure braid group $P_{n}$ is bi-orderable, and we will review the bi-order for the Thompson group $F$. Showing that there is a bi-order of $P_{n}$ which is consistent with the above direct system, we will be able to show that $P B V$ is bi-orderable. This fact, together with the bi-order of $F$, will allow us to show that $B F$ is also bi-orderable.

\section{ORDERINGS}

2.1. Concepts and basic properties. A group $G$ is said to be left-orderable if there exists a total order on its elements which is invariant under left multiplication, that is, $a<b$ implies $c a<c b$ for every $a, b, c \in G$ (see [14]). Such an order is determined by the set of positive elements, $P=\{x \in G ; 1<x\}$, since $a<b$ if and only if $1<a^{-1} b$, in other words, if $a^{-1} b \in P$.

Notice that every subset $P \in G$ determines a binary relation on the elements of $G$ in the above way $\left(a<b \Leftrightarrow a^{-1} b \in P\right)$. This relation is clearly invariant under left-multiplication. Moreover, the relation is transitive if and only if $P$ is a semigroup, and it is antisymmetric and total if and only if $G=P \sqcup\{1\} \sqcup P^{-1}$. Hence, a group $G$ is left-orderable if and only if it contains a subsemigroup $P \subset G$ such that $G=P \sqcup\{1\} \sqcup P^{-1}$.

A very simple example of left-orderable group is $\mathbb{Z}^{n}$, with the lexicographical order. A less obvious example is $B_{n}[8,10]$. Notice that, in a left-orderable group, all powers of a positive element are positive, hence left-orderable groups are torsion-free. This shows in particular that finite groups, as well as Thompson's group $V$, are not left-orderable.

If a group is left-orderable, one can use the monoid $P$ to define a right-order $\prec$, that is a total order of its elements which is invariant under right multiplication: we just say that $a \prec b$ if $b a^{-1} \in P$. Hence, a group is left-orderable if and only if it is right-orderable, but the two orderings do not necessarily coincide.

A group is said to be bi-orderable if it admits a left-order which is also a right-order. Notice that a group $G$ is bi-orderable if and only if it admits a subsemigroup $P$ such that $G=P \sqcup\{1\} \sqcup P^{-1}$ (thus $G$ is left-orderable), and furthermore $P$ is closed under conjugation in $G$ (thus the left-order is also a right-order). The group $\mathbb{Z}^{n}$ is hence biorderable, with the lexicographical order. The free group $F_{n}$ of rank $n$ is also bi-orderable, as we shall see later.

Notice that, in a bi-orderable group, every conjugate of a positive element is positive. Since products of positive elements are positive, this implies that a bi-orderable group cannot have generalized torsion, which means that the product of nontrivial conjugate elements can never be trivial. The braid group $B_{n}(n>2)$ is an example of a leftorderable group which is not bi-orderable, since it has generalized torsion. Namely, in $B_{n}$ one has $\sigma_{1} \sigma_{2}^{-1} \cdot\left(\sigma_{1} \sigma_{2} \sigma_{1}\right)^{-1} \sigma_{1} \sigma_{2}^{-1}\left(\sigma_{1} \sigma_{2} \sigma_{1}\right)=1$. But $B_{n}$ contains a finite index subgroup which is bi-orderable, namely the pure braid group $P_{n}$. Later in this section we will recall the explicit bi-order that can be defined in $P_{n}$.

There is a well-known result (see for instance [12]) that shows how left and bi-orderability behave under extensions.

Proposition 2.1. Consider the following exact sequence of groups:

$$
1 \longrightarrow A \stackrel{\alpha}{\longrightarrow} B \stackrel{\beta}{\longrightarrow} C \longrightarrow 1 \text {. }
$$


Suppose that $A$ and $C$ are left-orderable, and let $P_{A}$ and $P_{C}$ be their corresponding semigroups of positive elements. Then $B$ is also left-orderable, an explicit left-order being defined by the semigroup $\alpha\left(P_{A}\right) \cup \beta^{-1}\left(P_{C}\right)$.

Moreover, suppose that $P_{A}$ and $P_{C}$ define bi-orders on $A$ and $C$ respectively. Suppose also that the above sequence splits, so $B=C \ltimes A$. If the action of $C$ on $A$ preserves the order in $A$ (i.e. preserves $P_{A}$ ), then $B$ is bi-orderable, an explicit bi-order being defined by the semigroup $\alpha\left(P_{A}\right) \cup \beta^{-1}\left(P_{C}\right)$.

The above left-order (say bi-order) of $B$ can be explained as follows: An element $b \in B$ is positive if and only if $\beta(b)$ is positive in $C$, or $\beta(b)=1$ and $b$ is positive in $A$. In the case of the semi-direct product, we can just say that the order in $B=C \ltimes A$ is the lexicographical order.

2.2. Explicit bi-orders in some groups. We will now explain some specific bi-orders in three groups which are important for our purposes. They are Thompson's group $F$, the free group of rank $n, F_{n}$, and the pure braid group on $n$ strands $P_{n}$. The three groups are already known to be bi-orderable, and we shall explain the known bi-orders of $F_{n}$ and $P_{n}$, together with a review of the ordering of $F$.

The bi-order in $F$ is defined as follows. Recall that an element $f \in F$ is a piecewise-linear, orientation preserving homeomorphism of the interval $[0,1]$ and all slopes are powers of 2 . An element is then positive if its first slope different from 1 is a positive power of 2 . It is not difficult to prove that it is a bi-order, see [9] for details and other descriptions of this bi-order. It is important to realize that this definition of positive element is different from a commonly used notion of positive word in $F$, which is given by the appearance of only positive generators (no inverses) in the normal form for the element. In this paper, the word "positive", referring to elements of $F$, will always make reference to the definition above, that is, their first nontrivial slope being a positive power of 2 .

We will now recall the usual bi-order of the free group $F_{n}$. A detailed proof can be found in [12]. It is based on the so called Magnus expansion of the free group [13], so it is usually called the Magnus ordering. Let $F_{n}$ be the free group of rank $n$, freely generated by $x_{1}, \ldots, x_{n}$. Let $\mathbb{Z}[[\mathbf{X}]]=\mathbb{Z}\left[\left[X_{1}, \ldots, X_{n}\right]\right]$ be the ring of formal series on $n$ non-commutative variables $X_{1}, \ldots, X_{n}$, with coefficients in $\mathbb{Z}$. The Magnus expansion is the homomorphism $\varphi: F_{n} \longrightarrow \mathbb{Z}[[\mathbf{X}]]$ defined by $\varphi\left(x_{i}\right)=1+X_{i}$, for $i=1, \ldots, n$. Notice that one then has $\varphi\left(x_{i}^{-1}\right)=1-X_{i}+X_{i}^{2}-X_{i}^{3}+\cdots$. It is shown in [13] that $\varphi$ is injective. The elements in $\operatorname{Im}(\varphi)$ are formal series $f(\mathbf{X})$ such that $f(\mathbf{0})=1$.

Notice that the set of monomials of $\mathbb{Z}[[\mathbf{X}]]$ can be totally ordered: First, we order the variables by $X_{1}<X_{2}<\cdots<X_{n}$. Then, given two monomials, the smallest one will be the one of smallest degree, or in case their degrees coincide, the smallest one in lexicographical order. One can then say that a nontrivial element $x \in F_{n}$ is positive if the coefficient of the smallest nontrivial term of $\varphi(x)-1$ is positive. This defines a set $P$ of positive elements in $F_{n}$ which yields a bi-order of $F_{n}$.

There is an important property of the Magnus expansion that we will need later. Define the map $\delta: F_{n} \rightarrow \mathbb{Z}[[\mathbf{X}]]$ to be the map (but not homomorphism) that sends the trivial element to 0 , and every nontrivial $x \in F_{n}$ to the nontrivial homogeneous form of smallest degree in $\varphi(x)-1$. The form $\delta(x)$ is called the deviation of $x$. Notice that $x$ is positive if and only if the coefficient of the smallest term in $\delta(x)$ is positive. Consider now the 
lower central series of $F_{n}, F_{n}=G_{1} \supset G_{2} \supset G_{3} \supset \cdots$. It is shown in [13] that $\delta(x)$ has degree $d$ if and only if $x \in G_{d}$ and $x \notin G_{d+1}$. Moreover, if we denote $\mathbb{Z}[[\mathbf{X}]]_{d}$ the set of homogeneous forms of degree $d$ in $\mathbb{Z}[[\mathbf{X}]]$, one has:

Theorem 2.2. [13] For every $d>0$, the map $\delta$ determines a one-to-one homomorphism from the abelian group $G_{d} / G_{d+1}$, under group multiplication, to $\mathbb{Z}[[\mathbf{X}]]_{d}$, under addition.

We end this section with a exposition of a bi-order of the pure braid group $P_{n}$, given in [12]. It comes from the above bi-orders of free groups, together with Proposition 2.1, since there is a well-known split exact sequence of groups:

$$
1 \longrightarrow F_{n-1} \longrightarrow P_{n} \stackrel{\eta}{\longrightarrow} P_{n-1} \longrightarrow 1,
$$

where for every braid $p \in P_{n}, \eta(p)$ is the braid obtained from $p$ by deleting its first strand. Indeed, if all strands except the first one are trivial in a pure braid $p$, these trivial strands can be considered as being punctures of the plane, and the first strand of $p$ can be considered as describing a loop in the $(n-1)$-punctured plane. Hence ker $\eta$ is isomorphic to the fundamental group of the $(n-1)$-punctured plane, which is a free group on $n-1$ generators. The above sequence clearly splits, by adding a strand to the left of a braid in $P_{n-1}$, so $P_{n}=P_{n-1} \ltimes F_{n-1}$, and the action of $P_{n-1}$ on $F_{n-1}$ preserves the Magnus ordering of $F_{n-1}[12]$. It follows by recurrence on $n$ that $P_{n}=\left(\cdots\left(\left(F_{1} \ltimes F_{2}\right) \ltimes F_{3}\right) \ltimes \cdots F_{n-2}\right) \ltimes F_{n-1}$, and $P_{n}$ is bi-orderable by Proposition 2.1, an explicit bi-order being the lexicographical order in $\left(\cdots\left(\left(F_{1} \ltimes F_{2}\right) \ltimes F_{3}\right) \ltimes \cdots F_{n-2}\right) \ltimes F_{n-1}$, using the Magnus ordering in each $F_{i}$. This semidirect product decomposition is called the Artin combing of $P_{n}$.

It will be convenient to introduce a free generating set for each $F_{i}$ in the above semidirect product decomposition of $P_{n}$. Notice that each element in $F_{i}$ corresponds to a loop made by the strand $n-i$ crossing the strands $n-i+1, n-i+2, \ldots, n$. In this way, if we consider the pure braid $A_{i, j}$ in the left hand side of Figure 6 , the group $F_{i}$ is freely generated by $\left\{A_{n-i, j} ; j>n-i\right\}$. In the right hand side of Figure 6 we can see the Artin combing of the pure braid $p$ in Figure 4 , which is $p=\left(A_{3,5}\right)\left(A_{2,3}^{-1}\right)\left(A_{1,3}^{-1} A_{1,2}^{-1} A_{1,3}\right)$. Notice that the strands 4 and 5 do not cross, so the first nontrivial factor in the decomposition of $p$ is $A_{3,5}$, which is a positive element of $F_{2}$. Hence $p$ is a positive pure braid.

\section{THE MAIN THEOREM}

We have already described all the tools we need to show the main results of this paper, namely that $P B V$ and $B F$ are bi-orderable. Recall that $P B V=\underset{T}{\lim _{\longrightarrow}} P_{n, T}$, that is, $P B V$ is the direct limit of an infinite number of copies of $P_{n}$ (with distinct values of $n$ ). We already know that each $P_{n}$ has a bi-order, hence $P_{n, T}$ is bi-orderable for every $T$, where $(T, p, T) \in P_{n, T}$ is said to be positive if and only if $p$ is a positive pure braid. But it is not clear that this order is compatible with the above direct system. This is shown in the following result, which we will use several times later.

Lemma 3.1. The element $(T, p, T) \in P_{n, T}$ is positive in $P_{n, T}$ if and only if $\alpha_{n, T, i}(T, p, T)$ is positive in $P_{n+1, T^{\prime}}$, for any $i=1, \ldots, n$.

Proof. Since the order in $P_{n}$ is determined by the Artin combing, we need to see how the Artin combing of a pure braid $p \in P_{n}$ is transformed when we apply $\alpha_{n, T, i}$ to the element $(T, p, T)$. Suppose that $p=f_{1} f_{2} \cdots f_{n-1}$ is the Artin combing of $p$, where each 


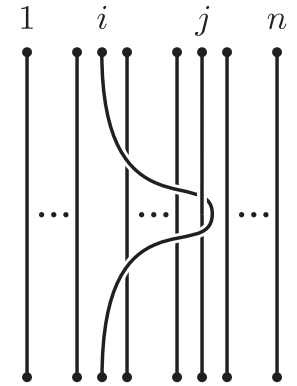

$A_{i, j}$

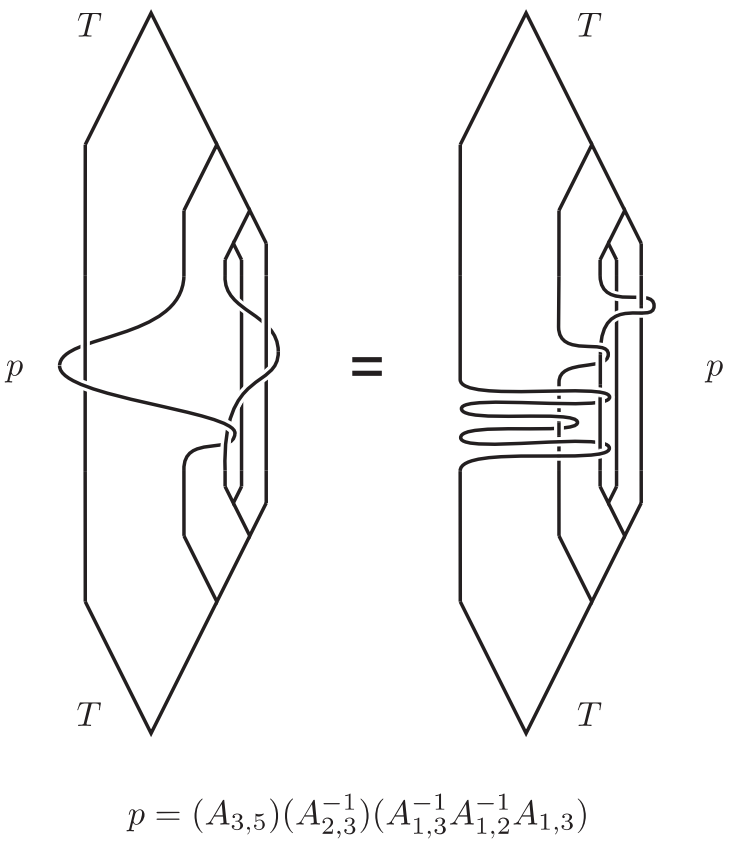

Figure 6 . The generators $A_{i, j}$, and the combing of the pure braid $p$.

$f_{i} \in F_{i}=\left\langle A_{n-i, l} ; l>n-i\right\rangle$, and suppose that $f_{j}$ is the first nontrivial factor in the above decomposition, for some $j \geq 1$. This means that the last $j$ strands of $p$ form a trivial braid, while the last $j+1$ strands do not. Now suppose we apply $\alpha_{n, T, i}$ to $(T, p, T)$. The pure braid $p$ is then replaced by $p^{\prime}$, which is obtained from $p$ by doubling its $i$-th strand. Let $p^{\prime}=f_{1}^{\prime} f_{2}^{\prime} \cdots f_{n}^{\prime}$ be the Artin combing of $p^{\prime}$ (notice that $p^{\prime}$ has one more strand, hence one more factor in its Artin combing). We will distinguish two cases.

If $i \leq n-j$, the last $j+1$ strands of $p$ and $p^{\prime}$ form the same braid, only that the indices in $p^{\prime}$ are shifted by one. Hence $f_{1}^{\prime}, \ldots, f_{j-1}^{\prime}$ are all trivial, and $f_{j}^{\prime}$ is obtained from $f_{j}$ by replacing each $A_{n-j, k}$ by $A_{n-j+1, k+1}$. Since $f_{j}$ and $f_{j}^{\prime}$ determine the same element of $F_{j}$, their Magnus expansions coincide, hence $p$ is positive if and only if $p^{\prime}$ is positive. The geometric meaning of this computation is that, in this case, after recombing the braid obtained by splitting the $i$-th strand, the first factor of the combing is the same as before, which is clear geometrically.

If $i>n-j$, we have doubled one of the last $j$ strands of $p$, hence $p^{\prime}$ is a braid whose last $j+1$ strands form a trivial braid, and then $f_{1}^{\prime}, \ldots, f_{j}^{\prime}$ are all trivial. Moreover, $f_{j+1}^{\prime}$ is obtained from $f_{j}$ by replacing each $A_{n-j, k}$ either by $A_{n-j, k}$ (if $k<i$ ), by $A_{n-j, k+1}$ (if $k>i$ ), or by $A_{n-j, k} A_{n-j, k+1}\left(\right.$ if $k=i$ ). To simplify notation, if we denote $A_{n-j, k}$ by $x_{k}$, then $f_{j+1}^{\prime}$ is obtained from $f_{j}$ by the injective homomorphism

$$
\theta_{i}: F_{n} \rightarrow F_{n+1}
$$

given by

$$
\theta_{i}\left(x_{k}\right)= \begin{cases}x_{k} & \text { if } k<i \\ x_{k} x_{k+1} & \text { if } k=i \\ x_{k+1} & \text { if } k>i\end{cases}
$$

The problem is now reduced to its algebraic setting. The proof will be finished if we show that $f \in F_{n}$ is positive if and only if $\theta_{i}(f) \in F_{n+1}$ is positive. We will do this 
by looking at the deviations of $f$ and $\theta_{i}(f)$. Let $F_{n}=G_{1} \supset G_{2} \supset G_{3} \supset \cdots$ and $F_{n+1}=G_{1}^{\prime} \supset G_{2}^{\prime} \supset G_{3}^{\prime} \supset \cdots$ be the lower central series of $F_{n}$ and $F_{n+1}$, respectively. Suppose that $f \in G_{d}$ and $f \notin G_{d+1}$, so its deviation $\delta(f)$ is a form of degree $d$ in $\mathbb{Z}[[\mathbf{X}]]$. It is well-known [13] that $G_{d} / G_{d+1}$ is generated by the elements $\left[\left[\cdots\left[\left[x_{i_{1}}, x_{i_{2}}\right], x_{i_{3}}\right], \cdots\right], x_{i_{d}}\right]$, where $i_{k} \in\{1, \ldots, n\}$ for $k=1, \ldots, d$, and $[a, b]=a^{-1} b^{-1} a b$. We can assume $i_{1} \neq i_{2}$, since otherwise the bracket is trivial. These generators are called simple commutators, and we remark that they do not form a basis of the abelian group $G_{d} / G_{d+1}$, although they do generate the group. As in [13], we will denote each of the above simple commutators by $\left[x_{i_{1}}, x_{i_{2}} \ldots, x_{i_{d}}\right]$.

It is shown in [13] that given $x, y \in F_{n}$ then, if $\delta(x) \delta(y)-\delta(y) \delta(x) \neq 0$, one has $\delta([x, y])=$ $\delta(x) \delta(y)-\delta(y) \delta(x)$. Hence, since $\delta\left(x_{k}\right)=X_{k}$ for $k=1, \ldots, n$, it follows that $\delta\left(\left[x_{i_{1}}, x_{i_{2}}\right]\right)=$ $X_{i_{1}} X_{i_{2}}-X_{i_{2}} X_{i_{1}}$, provided $i_{1} \neq i_{2}$. We will show by induction on $d$ that

$$
\delta\left(\left[x_{i_{1}}, \ldots, x_{i_{d}}\right]\right)=\delta\left(\left[x_{i_{1}}, \ldots, x_{i_{d-1}}\right]\right) X_{i_{d}}-X_{i_{d}} \delta\left(\left[x_{i_{1}}, \ldots, x_{i_{d-1}}\right]\right),
$$

that no monomial in the above expression has the form $X_{j}^{d}$, and that the coefficient of the smallest monomial is either 1 or -1 . We know the claim is true for $d=2$, so suppose it is true for $d-1$ and let $M$ be the smallest monomial of $\delta\left(\left[x_{i_{1}}, \ldots, x_{i_{d-1}}\right]\right)$. Notice that the smallest monomial of $\delta\left(\left[x_{i_{1}}, \ldots, x_{i_{d-1}}\right]\right) X_{i_{d}}$ is $M X_{i_{d}}$, and the smallest monomial of $X_{i_{d}} \delta\left(\left[x_{i_{1}}, \ldots, x_{i_{d-1}}\right]\right)$ is $X_{i_{d}} M$. These two monomials cannot coincide, since this would imply that $M=X_{i_{d}}^{d-1}$, which is not true by induction hypothesis. Hence, either $M X_{i_{d}}$ or $X_{i_{d}} M$ is the smallest monomial of $\delta\left(\left[x_{i_{1}}, \ldots, x_{i_{d-1}}\right]\right) X_{i_{d}}-X_{i_{d}} \delta\left(\left[x_{i_{1}}, \ldots, x_{i_{d-1}}\right]\right)$. Moreover, its coefficient will be \pm 1 , since the same happens for $M$ in $\delta\left(\left[x_{i_{1}}, \ldots, x_{i_{d-1}}\right]\right)$, by induction hypothesis. This in particular implies that

$$
\delta\left(\left[x_{i_{1}}, \ldots, x_{i_{d-1}}\right]\right) X_{i_{d}}-X_{i_{d}} \delta\left(\left[x_{i_{1}}, \ldots, x_{i_{d-1}}\right]\right) \neq 0,
$$

and since $\delta\left(x_{i_{d}}\right)=X_{i_{d}}$ it follows that $\delta\left(\left[x_{i_{1}}, \ldots, x_{i_{d}}\right]\right)$ is equal to the above expression, as we wanted to show. It is also clear that no monomial of $\delta\left(\left[x_{i_{1}}, \ldots, x_{i_{d}}\right]\right)$ can be a power of a variable, since this would imply that the same happens for some monomial of $\delta\left(\left[x_{i_{1}}, \ldots, x_{i_{d-1}}\right]\right)$. We have then shown the claim.

A particular consequence of the above claim is that

$$
\delta\left(\left[x_{i_{1}}, x_{i_{2}} \ldots, x_{i_{d}}\right]\right)=\sum_{\sigma \in \Sigma} \varepsilon_{\sigma} X_{i_{\sigma(1)}} X_{i_{\sigma(2)}} \cdots X_{i_{\sigma(d)}},
$$

where $\Sigma$ is a certain subset of the symmetric group $S_{d}$ (which only depends on $d$ ) and $\varepsilon_{\sigma}= \pm 1$. Hence, every monomial in $\delta\left(\left[x_{i_{1}}, x_{i_{2}} \ldots, x_{i_{d}}\right]\right)$ consists of a permutation of the variables. Notice that if there are some repeated variables in $X_{i_{1}}, \ldots, X_{i_{d}}$, then some monomials in the above expression may coincide, so the coefficients in the form $\delta\left(\left[x_{i_{1}}, x_{i_{2}} \ldots, x_{i_{d}}\right]\right)$ may be distinct from \pm 1 .

Since we will compare simple commutators in $F_{n}$ and in $F_{n+1}$, we will need the following concepts. Given a simple commutator $\left[x_{i_{1}}, \ldots, x_{i_{d}}\right] \in F_{n}$ and a simple commutator $\left[x_{j_{1}}, \ldots, x_{j_{d}}\right] \in F_{n+1}$, we will say that the latter is an $i$-successor of the former if it is obtained from it by replacing each $x_{k}$ by $x_{k+1}$, if $k>i$, and each $x_{i}$ by either $x_{i}$ or $x_{i+1}$. Notice that if $m$ is the number of appearances of $x_{i}$ in $\left[x_{i_{1}}, \ldots, x_{i_{d}}\right]$, then this commutator has $2^{m} i$-successors. In the same way, if $M$ is a monomial in the variables $X_{1}, \ldots, X_{n}$, and $M^{\prime}$ is a monomial in the variables $X_{1}, \ldots, X_{n+1}$, we will say that $M^{\prime}$ is an $i$-successor of $M$ if it is obtained from it by replacing each $X_{k}(k>i)$ by $X_{k+1}$ and each $X_{i}$ by either $X_{i}$ or $X_{i+1}$. As above, if $m$ is the number of appearances of $X_{i}$ in $M$, then $M$ has $2^{m}$ $i$-successors. Notice also that among those $2^{m} i$-successors, there is only one which does 
not involve the variable $X_{i+1}$, which we will call the minimal $i$-successor of $M$, since it is the smallest one with respect to the total order on monomials defined above.

Let us define $\xi_{i}: \mathbb{Z}\left[\left[X_{1}, \ldots, X_{n}\right]\right] \rightarrow \mathbb{Z}\left[\left[X_{1}, \ldots, X_{n+1}\right]\right]$ to be the ring homomorphism given by

$$
\xi_{i}\left(X_{k}\right)= \begin{cases}X_{k} & \text { if } k<i \\ X_{k}+X_{k+1} & \text { if } k=i \\ X_{k+1} & \text { if } k>i\end{cases}
$$

Observe that if $M$ is a monomial in the variables $X_{1}, \ldots, X_{n}$, then $\xi_{i}(M)$ is equal to the sum of all $i$-successors of $M$.

Now suppose we apply $\theta_{i}$ to $\left[x_{i_{1}}, \ldots, x_{i_{d}}\right]$. This would replace each $x_{i_{k}}$ by either $x_{i_{k}}$ or $x_{i_{k}} x_{i_{k}+1}$ or $x_{i_{k}+1}$, depending wether $i_{k}$ is smaller than, equal to or greater than $i$, respectively. It is shown in [13] that for every $a_{1}, \ldots, a_{k-1}, a_{k}, a_{k}^{\prime}, a_{k+1}, \ldots, a_{d} \in F_{n+1}$ (actually in any group), one has $\left[a_{1}, \ldots, a_{k} a_{k}^{\prime}, \ldots, a_{d}\right]=\left[a_{1}, \ldots, a_{k}, \ldots, a_{d}\right]\left[a_{1}, \ldots, a_{k}^{\prime}, \ldots, a_{d}\right](\bmod$ $\left.G_{d+1}^{\prime}\right)$. Hence, if we define $\mathcal{S}$ to be the set of all $i$-successors of $\left[x_{i_{1}}, \ldots, x_{i_{d}}\right]$, one has

$$
\theta_{i}\left(\left[x_{i_{1}}, \ldots, x_{i_{d}}\right]\right)=\prod_{\left[x_{j_{1}}, \ldots, x_{j_{d}}\right] \in \mathcal{S}}\left[x_{j_{1}}, \ldots, x_{j_{d}}\right] \quad\left(\bmod G_{d+1}^{\prime}\right)
$$

Now, if we apply $\delta$ to $\theta_{i}\left(\left[x_{i_{1}}, \ldots, x_{i_{d}}\right]\right)$, Theorem 2.2 tells us that

$$
\delta\left(\theta_{i}\left(\left[x_{i_{1}}, \ldots, x_{i_{d}}\right]\right)\right)=\sum_{\left[x_{j_{1}}, \ldots, x_{j_{d}}\right] \in \mathcal{S}} \delta\left(\left[x_{j_{1}}, \ldots, x_{j_{d}}\right]\right) .
$$

Let us see that the above form can be rewritten as follows:

$$
\delta\left(\theta_{i}\left(\left[x_{i_{1}}, \ldots, x_{i_{d}}\right]\right)\right)=\xi_{i}\left(\delta\left(\left[x_{i_{1}}, \ldots, x_{i_{d}}\right]\right)\right) .
$$

Indeed, by (2) and (3) one has

$$
\begin{gathered}
\delta\left(\theta_{i}\left(\left[x_{i_{1}}, \ldots, x_{i_{d}}\right]\right)\right)=\sum_{\left[x_{j_{1}}, \ldots, x_{j_{d}}\right] \in \mathcal{S}}\left(\sum_{\sigma \in \Sigma} \varepsilon_{\sigma} X_{j_{\sigma(1)}} X_{j_{\sigma(2)}} \cdots X_{j_{\sigma(d)}}\right) \\
=\sum_{\sigma \in \Sigma} \varepsilon_{\sigma}\left(\sum_{\left[x_{j_{1}}, \ldots, x_{j_{d}}\right] \in \mathcal{S}} X_{j_{\sigma(1)}} X_{j_{\sigma(2)}} \cdots X_{j_{\sigma(d)}}\right) \\
=\sum_{\sigma \in \Sigma} \varepsilon_{\sigma} \xi_{i}\left(X_{i_{\sigma(1)}} X_{i_{\sigma(2)}} \cdots X_{i_{\sigma(d)}}\right) \\
=\xi_{i}\left(\sum_{\sigma \in \Sigma} \varepsilon_{\sigma} X_{i_{\sigma(1)}} X_{i_{\sigma(2)}} \cdots X_{i_{\sigma(d)}}\right) \\
=\xi_{i}\left(\delta\left(\left[x_{i_{1}}, \ldots, x_{i_{d}}\right]\right)\right) .
\end{gathered}
$$

Finally, suppose that $f \in F_{n}$ belongs to $G_{d}$ but not to $G_{d+1}$. Since the set of simple commutators generates $G_{d} / G_{d+1}$, one has $f=c_{1}^{e_{1}} \cdots c_{t}^{e_{t}}\left(\bmod G_{d+1}\right)$, where each $c_{j}$ is a simple commutator of order $d$. By Theorem 2.2 and by the fact that $\theta_{i}$ and $\xi_{i}$ are homomorphisms, it follows that $\delta\left(\theta_{i}(f)\right)=\xi_{i}(\delta(f))$, since the same equality is true for the generators $c_{j}$.

Therefore, every monomial in $\delta\left(\theta_{i}(f)\right)$ with nontrivial coefficient is an $i$-successor of a unique monomial of $\delta(f)$, and furthermore their coefficients coincide. This means that the smallest monomial in $\delta\left(\theta_{i}(f)\right)$ is precisely the minimal $i$-successor of the smallest 
monomial of $\delta(f)$. Since their coefficients coincide, it follows that $f$ is positive if and only if so is $\theta_{i}(f)$, as we wanted to show. The proof of Lemma 3.1 is finished.

From Lemma 3.1 we see that doubling the $i$-th strand preserves the order of the pure braid group, so $\alpha_{n, T, i}$ preserves the order of $P_{n, T}$. It follows that if $(T, p, T)$ and $\left(T^{\prime}, p^{\prime}, T^{\prime}\right)$ are two representatives of the same element in $P B V$, then $p$ is a positive pure braid if and only if so is $p^{\prime}$. This allows to define an ordering in $P B V$, just by saying that $(T, p, T)$ is positive if and only if $p$ is a positive pure braid. By Lemma 3.1 this is well defined, and by the following result, it is a bi-order.

Corollary 3.2. The group PBV is bi-orderable.

Proof. Since $P_{n}$ is bi-orderable for every $n$, and $P_{n}$ is isomorphic to $P_{n, T}$, we can define $\mathcal{P}_{n, T}$ to be the semigroup of positive elements of $P_{n, T}$, that is $\mathcal{P}_{n, T}=\left\{(T, p, T) \in P_{n, T} ; 1<p\right\}$. Now define $\mathcal{P}=\underset{T}{\lim } \mathcal{P}_{n, T} \subset P B V$, that is, $\mathcal{P}$ is the set of elements in $P B V$ having one representative $(T, p, T)$ such that $p$ is positive. By Lemma 3.1, $\mathcal{P}$ is also the set of elements in $P B V$ all of whose representatives have the form $(T, p, T)$ with $p$ a positive braid. We will show that this set defines a bi-order in $P B V$. We must show that $\mathcal{P}$ is a semigroup, that $P B V=\mathcal{P} \sqcup\{1\} \sqcup \mathcal{P}$, and that $\mathcal{P}$ is invariant under conjugation.

We see that $\mathcal{P}$ is a semigroup, since two elements $\left(T_{1}, p_{1}, T_{1}\right),\left(T_{2}, p_{2}, T_{2}\right) \in \mathcal{P}$ can be multiplied by finding suitable representatives with matching trees, say $\left(T, p_{1}^{\prime}, T\right),\left(T, p_{2}^{\prime}, T\right)$, and their product will be $\left(T, p_{1}^{\prime} p_{2}^{\prime}, T\right)$. Since $p_{1}$ and $p_{2}$ are positive pure braids, from Lemma 3.1, the same happens to $p_{1}^{\prime}$ and $p_{2}^{\prime}$, hence $p_{1}^{\prime} p_{2}^{\prime}$ is also positive, and so is $\left(T, p_{1}^{\prime} p_{2}^{\prime}, T\right) \in \mathcal{P}$. On the other hand, since the inverse of $(T, p, T) \in P B V$ is $\left(T, p^{-1}, T\right)$, it follows immediately that $P B V=\mathcal{P} \sqcup\{1\} \sqcup \mathcal{P}^{-1}$. Finally, the action of conjugating an element $\left(T_{1}, p_{1}, T_{1}\right) \in \mathcal{P}$ by another element $\left(T_{2}, p_{2}, T_{2}\right) \in P B V$ is done by obtaining suitable representatives $\left(T, p_{1}^{\prime}, T\right) \in \mathcal{P}$ and $\left(T, p_{2}^{\prime}, T\right) \in P B V$, and the result is $\left(T, p_{2}^{\prime} p_{1}^{\prime}\left(p_{2}^{\prime}\right)^{-1}, T\right)$. Since the set of positive pure braids is invariant under conjugation, it follows that the resulting pure braid is positive, so $\mathcal{P}$ is invariant under conjugation. Therefore $\mathcal{P}$ defines a bi-order on $P B V$, as we wanted to show.

Theorem 3.3. The group BF is bi-orderable.

Proof. This is a consequence of the above corollary, together with Proposition 2.1, since by definition of $B F$ one has a short exact sequence

$$
1 \longrightarrow P B V \longrightarrow B F \stackrel{\widetilde{\rho}}{\longrightarrow} F \longrightarrow 1 \text {. }
$$

Moreover, this sequence splits, a section of $\widetilde{\rho}$ being the map that sends $\left(T_{-}, T_{+}\right) \in F$ to $\left(T_{-}, 1, T_{+}\right) \in B F$. Hence $B F=F \ltimes P B V$. By Proposition 2.1, we just need to show that the action of $F$ on $P B V$ preserves the order of $P B V$ defined in Corollary 3.2. The action determined by an element $\left(T_{-}, T_{+}\right) \in F$ on an element $(T, p, T) \in$ $P B V$ is given by conjugating the latter by $\left(T_{-}, 1, T_{+}\right)$, that is, if we choose representatives $\left(T_{-}^{\prime}, 1, T^{\prime}\right)=\left(T_{-}, 1, T_{+}\right)$and $\left(T^{\prime}, p^{\prime}, T^{\prime}\right)=(T, p, T)$, then the conjugate is $\left(T_{-}^{\prime}, 1, T^{\prime}\right)\left(T^{\prime}, p^{\prime}, T^{\prime}\right)\left(T^{\prime}, 1, T_{-}^{\prime}\right)=\left(T_{-}^{\prime}, p^{\prime}, T_{-}^{\prime}\right)$. Hence the pure braid $p$ is replaced by $p^{\prime}$. Since $(T, p, T)=\left(T^{\prime}, p^{\prime}, T^{\prime}\right)$, by Lemma 3.1, it follows that $p$ is positive if and only if so is $p^{\prime}$. Therefore, the action of $F$ on $P B V$ preserves the order, so $B F$ is bi-orderable as we wanted to show, an explicit bi-order being the lexicographic order in $F \ltimes P B V$, where $F$ is given the usual bi-order and $P B V$ is bi-ordered as in Corollary 3.2. 
Notice that the order in $B F$ can be easily described. An element $\left(T_{-}, p, T_{+}\right)$is positive if $\left(T_{-}, T_{+}\right) \in F$ is positive (its first slope different from 1 is greater than one), or if $\left(T_{-}, T_{+}\right)$ is trivial $\left(T_{-}=T_{+}\right)$and $p$ is a positive pure braid.

\section{REFERENCES}

[1] E. Artin, Theory of braids, Annals of Math. 48 (1946) 101-126.

[2] J. S. Birman, Braids, Links, and Mapping Class Groups. Annals of Math. Studies, 82, Princeton University Press 1975.

[3] J. S. Birman, T.E. Brendle, Braids: A Survey. www.arxiv.org/math.GR/0409205

[4] T. Brady, J. Burillo, S. Cleary and M. Stein, Pure braid subgroups of braided Thompson's groups, www.arxiv.org/math.GR/0603548

[5] M. G. Brin, The Algebra of Strand Splitting. I. A Braided Version of Thompson's Group V. www.arxiv.org/math.GR/0406042

[6] R. Geoghegan, K. S. Brown, An infinite-dimensional torsion-free $F P_{\infty}$ group, Invent. Math. 77 (1984) pp- 367-381.

[7] J. W. Cannon, W. J. Floyd and W. R. Parry, Introductory notes on Richard Thompson's groups. Enseign. Math. (2), 42 (3-4):215-256, 1996.

[8] P. Dehornoy, From large cardinals to braids via distributive algebra, J. Knot Theory Ramifications 4 (1995), no. 1, 33-79.

[9] P. Dehornoy, The group of parenthesized braids, Adv. in Math., to appear.

[10] R. Fenn, M. T. Greene, D. Rolfsen, C. Rourke and B. Wiest, Ordering the braid groups, Pacific J. Math. 191 (1999), no. 1, 49-74.

[11] V. L. Hansen, Braids and Coverings, Cambridge University Press, 1989, London Mathematical Society Student Texts, 18.

[12] D. M. Kim and D. Rolfsen, An ordering for groups of pure braids and fiber-type hyperplane arrangements. Canad. J. Math. 55, no. 4 (2003), 822-838.

[13] W. Magnus, Karras and Solitar, Combinatorial Group Theory. Presentations of groups in terms of generators and relations, Revised ed., Dover Publications, Inc., New York, 1976.

[14] R. Mura and A. Rhemtulla, Orderable Groups. Lecture Notes in Pure and Appl. Math. 27, Marcel Dekker, New York, 1977.

Departament de Matemática Aplicada IV, Escola Politècnica Superior de Castelldefels, Universitat Politécnica de Catalunya, Av. del Canal Olímpic s/n, 08860 Castelldefels, BARCElona, SpAin.

E-mail address: burillo@ma4.upc.edu

Departamento de Álgebra, Facultad de Matemáticas, Universidad de Sevilla, Apdo. 1160, 41080 SEVILla, SPAiN.

E-mail address: meneses@us.es 